\title{
Ultrafast polariton dynamics in strongly coupled zinc porphyrin microcavities at room temperature
}

\author{
P. G. Savvidis, ${ }^{1,2}$ L. G. Connolly, ${ }^{3}$ M. S. Skolnick, ${ }^{3}$ D. G. Lidzey, ${ }^{3}$ and J. J. Baumberg ${ }^{1}$ \\ ${ }^{1}$ Department of Physics \& Astronomy, University of Southampton, SO17 1BJ, United Kingdom \\ ${ }^{2}$ Department of Materials Science and Technology, University of Crete, and FORTH, P.O. 1527, 71110 Heraklion, Greece \\ ${ }^{3}$ Department of Physics and Astronomy, University of Sheffield, Sheffield S3 7RH, United Kingdom
}

(Received 20 June 2006; published 27 September 2006)

\begin{abstract}
Time and angle-resolved measurements reveal ultrafast dynamics of excitations in organic microcavities leading to dramatic modulation of probe transmission $(\sim 30 \%)$. We find that the induced changes have both fast and long-lived components. Fast response times are defined by vibronic relaxation and intersystem crossing $\left(S_{1} \rightarrow T_{1}\right)$, whereas long-lived changes are attributed to a build up of carriers in the nonradiative triplet state whose lifetime is longer than the repetition rate of the laser pulses, resulting thus in incomplete recovery of the ground state. Blueshifts of the lower polariton branch in the presence of the pump pulse indicate the presence of nonlinear interactions in the sample. However, there is no evidence yet for the pair-type scattering processes in porphyrin microcavities.
\end{abstract}

DOI: 10.1103/PhysRevB.74.113312

PACS number(s): 78.47.+p, 71.36.+c, 72.80.Le, 82.53.Xa

The modified dispersion that accompanies strong coupling of embedded resonant absorbers in an optical microcavity ${ }^{1}$ has been shown to introduce completely new and extremely strong nonlinear optical interactions. ${ }^{2-5}$ This has been shown in a wide variety of ways in conventional III- $\mathrm{V}$ heterostructure microcavities grown using traditional semiconductor fabrication. In particular, the polaritons on the lower branch of the dispersion relation can undergo mutual pair scattering allowed by energy and momentum conservation. ${ }^{6}$ The polariton-polariton interactions are made much stronger by their Bosonic character: the scattering rate into a final state is enhanced by occupation of that final state. This gives rise to ultrafast (ps) gain, up to $10^{8} / \mathrm{cm}$, parametric amplification of signal and idler, ultralow threshold lasing, Bose condensation of polaritons, ${ }^{7}$ and micron-long optical parametric oscillators. ${ }^{8}$ However, a number of practical problems prevent the simple implementation of these advantageous characteristics, the most critical of which is that the gain only exists below temperatures comparable to the light-matter coupling strength in the microcavity. ${ }^{9}$ Organic microcavities which use organic semiconductors in a more conventional mirror sandwich structure exhibit strong coupling at room temperature. ${ }^{10-12}$ Hence they are potentially promising candidates for the new parametric scattering regime at room temperature. ${ }^{13}$

In this work, we report on the initial study of excitonpolariton dynamics in strongly coupled organic microcavities filled with porphyrin based dye. Ultrafast changes in the transmitted probe pulse are observed when resonantly injecting polaritons at different points of the lower polariton brunch with short pump pulses. The changes in transmission have both short- and long-lived components. We attribute the short-lived component to fast vibronic relaxation $\left(\tau_{S 2}\right.$ $\sim$ few ps) of the excited $S_{2}$ state into the lower-lying $S_{1}$ state, then followed by intersystem crossing (ISC) $S_{1} \rightarrow T_{1}$ into triplet state $\left(\tau_{I S C} \sim 100 \mathrm{ps}\right)$, Fig. 1 . Notably ISC dominates over ground-state $S_{1} \rightarrow S_{0}$ relaxation because of typically long radiative lifetimes of the $S_{1}$ state of the order of $\tau_{S 1}$ $\sim$ few ns. Accumulation of carriers in the very slowly decay- ing $\left(\tau_{T 1} \sim 10 \mu \mathrm{s}\right)$ nonradiative triplet states is responsible for the observed long-lived response. Contrary to demonstrations of polariton amplification in III-V microcavities, our experiments show reduction in the transmitted probe power indicating that no parametric scattering is observed in these samples. Our experiments clearly suggest that new organic microcavity systems that do not have fast deactivation pathways to lower-lying states are required in order for polaritonpolariton interaction (parametric scattering of polaritons) to take place.

The microcavity (MC) under study consists of 125-nm-thick polysterene film doped with the molecular dye tetaphenyl-porphyrin zinc (ZnTPP) and positioned between two dielectric distributed Bragg reflector (DBR) mirrors. ${ }^{14}$ Organic film was spin-cast onto the PECVD grown DBR mirror composed of $11 \lambda / 4$ pairs of $\mathrm{SiN} / \mathrm{SiO}_{2}$ on a quartz substrate. The top DBR mirror consisting of $12 \lambda / 4$ layers of $\mathrm{TeO}_{2}$ and $\mathrm{LiF}$ was thermally evaporated onto the organic film to avoid damage to the film. The resulting cavity finesse was in excess of $Q>400$. The absorption spectra of the dye



FIG. 1. Absorption spectra of zinc porphyrin dye and corresponding energy states $S_{n}$ singlets, $T_{n}$ triplets. Microcavity is formed by positioning the organic film between two dielectric mirrors. Two new states LP, UP separated by Rabi energy are formed through resonant light-matter interaction. 



FIG. 2. (a) Angular dispersion of porphyrin microcavity, and pump and probe transmitted spectra at the angles indicated. (b) Transmitted probe spectra (resonantly tuned to the lower polariton dispersion) without the pump pulse and with pump pulse at time delays $-10,-5,0,1,3,5,10,20$ ps. Inset: time-resolved probe transmission at $2.83 \mathrm{eV}$.

is plotted in Fig. 1 showing peak absorbance at $2.76 \mathrm{eV}$ which corresponds to the $S_{2}$ singlet excited state. Resonantly coupling this transition to the MC confined photon produces two new eigenstates of the system, namely the lower and upper polariton modes, Fig. 1 (dashed). Continuous wave $(\mathrm{CW})$ characterization of these microcavities has been reported by Connolly et al. ${ }^{14}$ Here we map the microcavity dispersion relations by looking at transmission spectra at different angles using white light continuum from an optical parametric amplifier (OPA). In Fig. 2(a), both lower and upper polariton branches are clearly resolved, split by a Rabi energy of $135 \mathrm{meV}$.

Although it is possible to explore the nonlinearities using $\mathrm{CW}$ experiments, it has proved much more fruitful and clear to examine the nonlinear properties in the ultrafast regime, before combinations of different scatterings muddy the clear identification of the processes that occur. We have investigated the nonlinear response and the dynamics of the cavity polaritons, by employing angle resolved pump-probe experiments. This requires the use of two-color pump-probe ultrafast spectroscopy with the ability to tune the wavelength of each pulse independently in the 2.7-2.9-eV (460430-nm) spectral region. These are derived from an optical parametric amplifier (OPA) pumped by a regeneratively amplified Ti:sapphire system (RegA) producing $5 \mu \mathrm{J}, 150$-fs pulses at $50-250-\mathrm{kHz}$ rate. The frequency doubled pump is used to resonantly pump the lower polariton branch, whereas the probe pulse is produced by mixing the residual pump pulse at $800-860-\mathrm{nm}$ with the amplified idler pulse (around
$950 \mathrm{~nm}$ ) from the OPA, to generate tuneable pulses from $420-440 \mathrm{~nm}$. The tuning ranges were a little restricted by the available mixing processes. Despite this, they allowed us to investigate many of the expected parametric scattering regimes.

A strong 150-fs pump pulse excites the sample at the chosen angle of incidence tuned in wavelength to resonantly excite lower branch polaritons. The resonance condition is easily observed from an increase in the transmission through the sample, and a spectral hole eaten out of the reflection spectrum (corresponding to the lower polariton). A weaker probe pulse arrives at the sample at a different angle of incidence (using our ultrafast goniometer ${ }^{6}$ ), again resonantly tuned to the lower polariton, and the transmission of this probe pulse is measured in the presence and absence of the pump. The signature for polariton scattering is a pumpinduced change in probe transmission, which depends on the energy momentum $(E, k)$ of the initial and final polariton states.

We first present data with the pump pulse set to $\theta=40^{\circ}$ and the probe pulse set to $\theta=30^{\circ}$, injecting polaritons at a position from which pair scattering of pump polaritons would be possible. The polariton dispersion curve is shown in Fig. 2(a), together with spectra of transmitted pump and probe pulses at angles of $40^{\circ}$ and $30^{\circ}$, respectively. This shows the high quality of the sample polariton dispersion, and the independently tuned pump and probe pulses. Typical time-resolved data are shown in Fig. 2(b).

Injecting pump polaritons results in $\sim 30 \%$ reduction in probe transmission in comparison to the probe transmission in the absence of the pump, even for large negative time delays. This dramatic reduction contrasts with experiments on III-V microcavities which show an induced increase in probe transmission (as energy is transferred from pump to probe beams) at zero delay. Typical pump powers of sub$1 \mathrm{~W} / \mathrm{cm}^{2}$ were used, however, the changes here were linear in pump power with no threshold effect. The change occurs across the whole spectral bandwidth, and no clear spectral shift of the peak position is observed (indicating that heating of the sample is not severe). The time-resolved change in the probe transmission (inset) shows that the reduction in transmission reaches maximum modulation $\Delta T$ of $-5 \%$ within first $2 \mathrm{ps}$, decays slightly over $10 \mathrm{ps,}$, and then persists for long times $(\mu \mathrm{s})$. Notably, comparable modulation amplitudes have been reported for strongly coupled organic microcavities based on $J$ aggregates of cyanine dye, ${ }^{15}$ in which modulation amplitude was enhanced by more than one order of magnitude compared to bare organic film (no microcavity present). The presence of a long-lived signal is characteristic in metalloporphyrins because of the long lifetime of the first excited state and significant triplet intersystem crossing. This typically manifests itself as an incomplete recovery of the ground state because the lowest-lying triplet state becomes populated. ${ }^{16}$ The lifetime of these nonradiative triplet states is quite long (few tens of $\mu \mathrm{s}$ ), ${ }^{17-19}$ much longer than the repetition rate of the laser pulses $(40 \mathrm{kHz})$.

Similar results were obtained over a range of pump and probe angles. They suggest that parametric scattering is not observed in these samples. On the other hand, the observed changes indicate a rapid bleaching of the $S_{2}$ state after exci- 



FIG. 3. (a) Pumping away from the magic angle into the excitons. (b) Transmitted probe spectra without the pump pulse and with pump pulse at time delays $-10,-5,0,1,3,5,10,20$ ps. Inset: time-resolved probe transmission.

tation, which recovers slightly. This may be due to transfer to the $T_{1}, S_{1}$ states on a $10-50$-ps time scale. Further information can be gained from exciting the sample at higher energy into the bottleneck region of polariton dispersion (almost resonant to the $S_{2}$ exciton) as in Fig. 3(a).

In this case, we see similar results, with a decrease in probe transmission, which is larger in magnitude than when pumping at lower angles and energies. This may be due to excited excitons that remain for a longer time in the sample and are available to scatter to lower energy states. We also observe an ultrafast blueshift of the resonant polariton energy when the pump pulse is present, due to nonlinear interactions in the sample. ${ }^{21}$ Notably, the increased transmission around $2.8 \mathrm{eV}(450 \mathrm{~nm})$ is associated with the photobleaching of the Soret transition $\left(S_{0} \rightarrow S_{2}\right)$ also observed by O'Keefe et al. ${ }^{20}$ Again there is an ultrafast turn-on of the induced change, which decays on the same time scales noted previously. This leads us to believe that we excite real excitations in the molecules, which decay from the $S_{2}$ state into a long-lived state over a relatively short time scale $(\sim 100 \mathrm{ps})$, most likely the $T_{1}$ state which is metastable. The occupation of this state causes a change in the oscillator strength and energy position of the $S_{2}$ state, leading to the induced changes observed.

In summary, there is no evidence yet for pair-type scattering processes in porphyrin strongly coupled microcavities at $300 \mathrm{~K}$. Such processes are driven by exciton-exciton exchange interactions, for which there is preliminary evidence in our experiments. However, a small size of the excitons in these organic semiconductors reduces exciton interactions substantially leading to higher thresholds for pair scattering. It is also possible that due to low-lying metastable states which compete rapidly for the excitations injected, the pair scattering appears to be masked. On the other hand, other systems such as strong-coupled $J$-aggregate microcavities are much more promising for observation of stimulated scattering, since relaxation to lower-lying states in this material is strongly suppressed. The effect of injecting excitations into these molecules is as yet substantially unclear.

We enthusiastically acknowledge discussions with C.E. Finlayson and P.G. Lagoudakis. This work was partly supported by UK EPSRC GR/S09838, GR/S05687/01, Greek Ministry of Education PYTHAGORAS II GR/N 2099, and via EU 5th Framework research networks "ClermontII," "Stimscat," "HYTEC" (HPRN-CT-2002-00315).
${ }^{1}$ C. Weisbuch, M. Nishioka, A. Ishikawa, and Y. Arakawa, Phys. Rev. Lett. 69, 3314 (1992).

${ }^{2}$ P. Senellart and J. Bloch, Phys. Rev. Lett. 82, 1233 (1999).

${ }^{3}$ L. S. Dang et al., Phys. Rev. Lett. 81, 3920 (1998).

${ }^{4}$ A. I. Tartakovskii et al., Phys. Rev. B 62, R2283 (2000).

${ }^{5}$ D. Snoke, Science 298, 1368 (2002).

${ }^{6}$ P. G. Savvidis, J. J. Baumberg, R. M. Stevenson, M. S. Skolnick, D. M. Whittaker, and J. S. Roberts, Phys. Rev. Lett. 84, 1547 (2000).

${ }^{7}$ R. M. Stevenson et al., Phys. Rev. Lett. 85, 3680 (2000).

${ }^{8}$ J. J. Baumberg, P. G. Savvidis, R. M. Stevenson, A. I. Tartakovskii, M. S. Skolnick, D. M. Whittaker, and J. S. Roberts, Phys. Rev. B 62, R16247 (2000).

${ }^{9}$ M. Saba, C. Ciuti, J. Bloch, V. Thierry-Mieg, R. Andre, Le Si Dang, S. Kundermann, A. Mura, G. Bongiovanni, J. L. Staehli, and B. Deveaud, Nature (London) 414, 731 (2001).

${ }^{10}$ D. G. Lidzey, D. D. C. Bradley, T. Virgili, A. Armitage, M. S. Skolnick, and S. Walker, Phys. Rev. Lett. 82, 3316 (1999).

${ }^{11}$ D. G. Lidzey, D. D. C. Bradley, M. S. Skolnick, T. Virgili, S.
Walker, and D. M. Whittaker, Nature (London) 395, 53 (1998).

${ }^{12}$ J. R. Tischler, M. S. Bradley, V. Bulovic, J. H. Song, and A. Nurmikko, Phys. Rev. Lett. 95, 036401 (2005).

${ }^{13}$ Zoubi et al., Phys. Rev. B 72, 125306 (2005).

${ }^{14}$ L. G. Connolly, D. G. Lidzey, R. Butte, A. M. Adawi, D. M. Whittaker, M. S. Skolnick, and R. Airey, Appl. Phys. Lett. 83, 5377 (2003).

${ }^{15}$ J. H. Song, Y. He, A. V. Nurmikko, J. Tischler, and V. Bulovic, Phys. Rev. B 69, 235330 (2004).

${ }^{16}$ J. H. Brannon and D. Magde, J. Am. Chem. Soc. 102, 62 (1980).

${ }^{17}$ G. Maiya, A. Harriman, J. L. Sessler, G. Hemmi, T. Murai, and T. E. Mallouk, J. Phys. Chem. 93, 8111 (1989).

${ }^{18} \mathrm{Si}$, M. Yang, Y. Wang, L. Zhang, C. Li, D. Wang, S. Dong, and W. Sun, Opt. Commun. 109, 487 (1994).

${ }^{19}$ A. Harriman, B. G. Maiya, T. Murai, G. Hemmi, J. L. Sessler, and T. E. Mallouk, J. Chem. Soc., Chem. Commun. 89, 314 (1989).

${ }^{20}$ G. E. O'Keef, G. J. Denton, E. J. Harvey, R. T. Phillips, R. H. Friend, and H. L. Anderson, J. Chem. Phys. 104, 805 (1996).

${ }^{21}$ C. Ciuti et al., Phys. Rev. B 62, R4825 (2000). 